\title{
El liderazgo directivo en la gestión escolar. Comparación de casos
}

\author{
Ramona Isabel Ferreira \\ ramonaisabelferreira@gmail.com \\ Facultad de Humanidades y Ciencias de la Educación \\ Universidad Nacional de Pilar \\ Pilar - Paraguay
}

\section{RESUMEN}

La investigación denominada El liderazgo directivo en la gestión escolar. Comparación de casos, tiene como objetivo final describir, comparar y analizar artículos publicados en la web en relación al liderazgo directivo y su relación con la gestión escolar, para ello se define que es el líder, los tipos, así también que es el liderazgo y los tipos, además de narrar la relación en la gestión escolar de estos tipos de liderazgo. El artículo se realiza con la finalidad de cumplir el módulo de Taller de Elaboración de Artículos Científicos comprendido en el curso de Doctorado en Ciencias de la Educación, organizado por la Facultad de Humanidades y Ciencias de la Educación dependiente de la Universidad Nacional de Pilar. Se efectúa una exploración sistemática de artículos científicos publicados en relación al tema, para llegar a discusión y conclusión final.

Se enfatiza el papel del Liderazgo directivo en la Gestión educativa en relación con los resultados académicos, ya que, el buen desempeño de su Liderazgo pedagógico contribuye a la obtención de la Calidad educativa.

Palabras Claves: calidad educativa; liderazgo directivo; gestión educacional; análisis comparativo. 


\title{
Directive leadership in school management. Case comparison
}

\begin{abstract}
The research called Leadership leadership in school management. Comparison of cases, had as its final objective to describe, compare and analyze articles published on the web in relation to directive leadership and its relation to school management, for which it was defined that it is the leader, the types, as well as what is the leadership and the types, in addition to narrating the relationship in school management of these types of leadership.The article was carried out in order to complete the Scientific Articles Preparation Workshop module included the Doctorate course in Educational Sciences, organized by the Faculty of Humanities and Education Sciences dependent on the National University of Pilar. A systematic exploration of published scientific articles was carried out in relation to the subject, to reach discussion and final conclusion.

It emphasizes the role of Leadership in Educational Management in relation to academic results, since, the good performance of its Pedagogical Leadership contributes to the obtaining of Educational Quality.
\end{abstract}

Key Words: educational quality; executive leadership; educational management; comparative analysis

Artículo recibido: 10 Agosto. 2021 Aceptado para publicación: 07. Setiembre. 2021 Correspondencia: ramonaisabelferreira@gmail.com Conflictos de Interés: Ninguna que declarar 


\section{INTRODUCCIÓN}

El artículo se despliega con el objetivo de analizar El liderazgo directivo en la gestión escolar, considerando la importancia de la Gestión escolar y el liderazgo del Director para el logro de la calidad educativa. En efecto, lograr la calidad de la educación es trabajo de todos; sin embargo, recae en particular en el director de la escuela por ser el principal responsable del funcionamiento administrativo, comunitario, pedagógico y organizacional de la gestión educativa.

El trabajo se compone de teorías conceptuales de varios autores, así como las definiciones de líder, liderazgo, gestión escolar, tipos, características, etc, y su posterior análisis y reflexión del tema. La finalidad del artículo es realizar un análisis comparativo de las evidencias rescatadas de las literaturas internacionales en relación al tema.

La Gestión escolar de los directores de las instituciones educativas teniendo en cuenta el rol del director es clave en cuanto a crear las condiciones institucionales que promuevan la eficacia de la organización escolar, generando un clima escolar positivo, vinculando su quehacer con la mejora en los aprendizajes. Por lo mismo, la finalidad de este trabajo citado en el primer párrafo de la introducción.

\section{MÉTODO}

La investigación se desarrolló en el periodo comprendido de marzo a mayo del 2020. Se realizó una indagación sistemática de artículos científicos publicados en la web en relación al tema. Se encontraron una variedad de artículos en relación a la Gestión escolar y el tipo de liderazgo del Director; la metodología es descriptiva comparativa, puesto que se describe y compara la situación tal como fue, según los datos recolectados de varios autores.

\section{RESULTADOS}

Antes de exponer el tema principal es prioritario definir ciertos términos, como son líder y liderazgo, además de los tipos y estilos de liderazgo, esta previa exposición desde el punto de sita de varias fuentes.

Según el diccionario de la Real Academia Español, la palabra Liderazgo, significa Condición de líder o Ejercicio de las actividades del líder. Y la palabra líder que es oriunda del inglés leader 'guía', significa persona que dirige o conduce un partido político, un grupo social u otra colectividad. O, persona o entidad que va a la cabeza entre los de su clase, especialmente en una competición deportiva. 
Para Collao (1997), el líder es el conductor, guía, jefe, dirigente. Es el que va a la cabeza de la organización. Propone con claridad los medios o caminos para el logro de los objetivos y crea una visión de futuro al cual aspira llegar. (Idone, 2012)

Citando a Böhm et. al.at (2015), dicen que el líder transformacional se destaca por su alto estándar ético, cuestión que le hace ganar el respeto de sus seguidores y de la sociedad. En este contexto, la incorporación al interior del equipo de valores socialmente deseables tales como altruismo, conciencia, espíritu de cuerpo, cortesía y virtudes cívicas, Unidos a la credibilidad del líder conducen a la creación de un clima de altas exigencias morales. (Pedraja, Rodríguez, Araneda, \& Rodríguez, 2016)

La autoridad que tiene el líder de una organización no se mide por el tipo de poder que ejerce sobre los miembros de la misma, sino que se mide con base en su capacidad de hacer que los otros realicen acciones convencidos del objetivo que quieren lograr, convencidos de que su trabajo tiene un fin trascendente y no es sentido necesariamente como una obligación, además ésta se basa en el prestigio que ha logrado frente a sus seguidores, prestigio que ha ganado con el ejemplo y con su habilidad de empoderar a los demás en sus propias habilidades y talentos. (Purizaca, 2017)

Para Chiavenato (1999) Liderazgo es, además, la influencia interpersonal ejercida en una situación, dirigida a través del proceso de comunicación humana a la consecución de uno o diversos objetivos específicos. El liderazgo es considerado como un fenómeno que ocurre exclusivamente en la interacción social; debe ser analizado en función de las relaciones que existen entre las personas en una determinada estructura social, y no por el examen de una serie de características individuales. (Cultura, 2014)

Según Lussier y Achua (2002, p.6) se refiere al liderazgo como "un proceso en el cual influyen líderes sobre seguidores y viceversa, para lograr los objetivos de una organización a través del cambio". En este sentido, estos autores señalan que el liderazgo tiene una serie de elementos que deben ser considerados, tales como: influencia, líderes y seguidores, personas, cambio y objetivos organizacionales. (Cultura, 2014)

Rincón Serrano (2003), que expresa que el hecho de influir en otras personas y de que éstas te sigan, es ya una acción de liderazgo y viene a ser como el ejercicio de la autoridad, para que la gente, ya sea en grupo grande o pequeño, se oriente para conseguir una meta en común. Newstrom (2007, p. 196) afirma que "el liderazgo es el proceso que consiste 
en influir y apoyar a los demás para que lo sigan y se muestren con disposición para hacer todo aquello que es necesario". (Cultura, 2014)

En el liderazgo existen estilos que influyen en el desempeño del directivo respecto a la gestión que realiza en la organización. El término "estilo de liderazgo" se refiere el modo personal de actuar de un líder. El estilo de liderazgo se basa en la propia personalidad de cada individuo, en la forma de entender las relaciones en el trabajo, y los propios valores y principios éticos, es decir, es una forma compleja que define el tipo de comportamiento que cada persona lleva a cabo en sus diferentes facetas de su vida. (Reyes, Trejo, \& Topete, 2017)

Murillo (2016), señala dentro de los estudios que identifican los estilos de liderazgo en el ámbito de la educación, la propuesta de Kurt Lewin, la cual sigue vigente y establece tres estilos de liderazgo: el liderazgo autoritario, el liderazgo democrático y el liderazgo laissez faire. A continuación, se muestran en la tabla 1 las características de los principales estilos de liderazgo, en la cual se puede notar la tendencia del liderazgo hacia un enfoque más participativo, con énfasis en las relaciones interpersonales y la comunicación. Es importante señalar que los estilos de liderazgo no son excluyentes uno de otros, y que en la realidad no existen líderes que tengan un estilo puro, más bien predomina uno de ellos. (Reyes, Trejo, \& Topete, 2017)

Tabla 1. Características de los Estilos de liderazgo.

\begin{tabular}{|c|c|c|c|c|c|}
\hline Autoritario & Democrático & Laissez faire & Transaccional & Distribuido & Transformacional \\
\hline $\begin{array}{l}\text { Liderazgo } \\
\text { unidireccional. } \\
\text { Poder y toma de } \\
\text { decisiones } \\
\text { concentrados en } \\
\text { el lider. }\end{array}$ & $\begin{array}{l}\text { Colaboración y } \\
\text { participación } \\
\text { de los } \\
\text { miembros del } \\
\text { grupo. }\end{array}$ & $\begin{array}{l}\text { No ejerce su } \\
\text { función, no } \\
\text { se } \\
\text { responsabiliz } \\
\text { a del grupo. }\end{array}$ & $\begin{array}{l}\text { Proceso de } \\
\text { intercambio } \\
\text { entre líder y } \\
\text { seguidores. } \\
\text { Premio o } \\
\text { castigo en } \\
\text { función del } \\
\text { rendimiento. } \\
\text { Compuesto de } \\
\text { dos factores: } \\
\text { Recompensas } \\
\text { contingentes y } \\
\text { Dirección por } \\
\text { excepción. }\end{array}$ & $\begin{array}{l}\text { Participativo y } \\
\text { colegiado. } \\
\text { Otorga poder, } \\
\text { autonomía y } \\
\text { responsabilidad } \\
\text { a sus } \\
\text { colaboradores }\end{array}$ & $\begin{array}{l}\text { Participativo, } \\
\text { confianza en las } \\
\text { personas, } \\
\text { comunicación } \\
\text { interpersonal, criterios } \\
\text { de actuación basados } \\
\text { en la ética y principios } \\
\text { morales. } \\
\text { Líderes actúan como } \\
\text { agentes de cambio en } \\
\text { la organización. }\end{array}$ \\
\hline
\end{tabular}

También otras investigaciones desarrollan otros tipos de liderazgo: 
Liderazgo transformacional: El líder transformacional es aquel que se focaliza en el desarrollo y empoderamiento de sus seguidores, logrando además aumentar la motivación y la moral de éstos. Los líderes transformacionales generan propósitos y metas desafiantes por las cuales vale la pena esforzarse y logran movilizar a sus seguidores en procura de alcanzar tales propósitos y metas (Zhu et al., 2011). El liderazgo transformacional exhibe un conjunto de características: (Pedraja, Rodríguez, Araneda, \& Rodríguez, 2016)

- Influencia idealizada. Los líderes transformacionales son carismáticos, admirados, respetados, obtienen la confianza de la gente, quienes procuran imitarles (Mittal y Dhar, 2015).

- Motivación inspiracional. Se refiere al hecho de que los líderes transformacionales son capaces de motivar a los miembros de su equipo, proporcionando significado intrínseco a su trabajo; es decir, al percatarse de la relevancia de su trabajo no sólo para su equipo sino para la sociedad. Así se crea un ambiente de compromiso con la búsqueda de un futuro deseable y construible a partir del esfuerzo mancomunado (Chen et al., 2015).

- Estimulación intelectual. Los líderes transformacionales estimulan a sus seguidores a ser creativos e innovadores, cuestionando las formas tradicionales de resolver los problemas, y motivándoles a buscar por sí solos soluciones novedosas y superiores, en un marco de mejora e innovación continua (Yasin et al., 2014).

- Consideración individual. Los líderes transformacionales tienen un comportamiento de orientación, estímulo y apoyo a sus seguidores, con quienes actúan como mentor, fortaleciendo las relaciones interpersonales, distribuyendo responsabilidades y colaborando con ellos en su crecimiento personal y profesional (Avolio et al., 1999). El líder transformacional se destaca por su alto estándar ético, cues- tión que le hace ganar el respeto de sus seguidores y de la sociedad. En este con- texto, la incorporación al interior del equipo de valores socialmente deseables tales como altruismo, conciencia, espíritu de cuerpo, cortesía y virtudes cívicas, unidos a la credibilidad del líder, conducen a la creación de un clima de altas exigencias morales (Böhm et al., 2015).

Liderazgo transaccional: Este líder construye un acuerdo con sus seguidores respecto de las metas y las expectativas de recompensa; por lo cual, su accionar se focaliza en la medición y control de las bases convenidas, ya que si se logran las metas se generan las recompensas contingentes. Los términos del intercambio son más bien de corto plazo y 
el líder no procura cambios significativos en la cultura organizacional (Antonakis y House, 2014). (Pedraja, Rodríguez, Araneda, \& Rodríguez, 2016).

Estilo laissez faire: Este líder evita las responsabilidades y prefiere no tomar decisiones ni realizar acciones que le hagan responsable de los resultados. Así no sólo elude la responsabilidad, sino que reduce su autoridad 274 Revista de Pedagogía, vol. 37, no 100, 2016, pp. 269-287 dejando a los seguidores, grados máximos de libertad para actuar y resolver (Skogstad et al., 2014). (Pedraja R., Rodríguez P., Araneda G., \& Rodríguez M., 2016).

Liderazgo Educativo: las organizaciones educativas son los espacios donde se concreta el proceso enseñanza-aprendizaje que, además de desarrollar las capacidades cognitivas del estudiantado, potencializa la formación integral, donde la ética, estética y el componente cultural y espiritual subyacen en una relación armoniosa con un paradigma epistemológico responsable. Dada su complejidad, el liderazgo mediante el cual se gestan las organizaciones educativas debe trascender de un corte tradicional a un liderazgo renovador. En la actualidad: se evidencia que el desarrollo del liderazgo escolar es uno de varios factores indirectos que afectan el aprendizaje. El liderazgo va tomando posturas más descentralizadas, se evidencia la necesidad de estudiar el liderazgo tomando en cuenta elementos como el ejercicio del poder o el género del líder. (Acuña \& Bolívar, 2019)

Autores como. Nuraan Davids y Yusef Waghid (2019) afirman que este liderazgo escolar puede conceptualizarse como el conjunto de prácticas que despliegan los líderes de las instituciones escolares, que contribuyen a generar un sentido y visión compartida, que a su vez influye y moviliza el comportamiento de sus miembros hacia el alcance de los aprendizajes de los estudiantes y hacia el desempeño óptimo de los docentes en la sala de clases. Éste se trata de un efecto, que se ejerce indirectamente a través de la influencia que desarrollan los directivos para mejorar el trabajo que realizan los docentes en el aula Bolívar et. al (2013) al tocar el tema del liderazgo educativo, se sitúa en la preocupación común en los sistemas educativos sobre que se puede hacer, para dinamizar las escuelas, de modo que puedan conseguir buen aprendizaje para todos los alumnos, por lo tanto, va vinculada a la mejora educativa. (Acuña \& Bolívar, 2019).

De lo anterior se deduce que las instituciones deben rediseñar, nuevas estructuras que posibiliten dar respuestas a las nuevas tendencias del siglo XXI. Debido a que el 
liderazgo educativo es visto como un elemento clave para instaurar progreso, creando condiciones institucionales que hagan más fuertes a las organizaciones escolares, es importante velar y orientar hacia la calidad, es decir, una educación de alta productividad y un proceso continuo de mejoramiento. Un liderazgo educativo se caracteriza principalmente por una estimulación y no por una estipulación. Es decir, el liderazgo educativo te enseña y estimula a aprender, pero no te presenta qué y cómo debes hacerlo. Dicho de otra manera, el liderazgo educativo no te da un pez, te enseña a pescar. De ahí el nombre de liderazgo educativo y pedagógico por ello es de vital importancia que desde los primeros años se motive a que en los centros escolares se eduque desde un liderazgo educativo. ((Murillo et al., 2018). (Acuña \& Bolívar, 2019)

En síntesis, el liderazgo educativo se construye en la relación de todos los elementos expresados hasta ahora y en la interacción del sentido pedagógico con la innovación y la creatividad de sus líderes y la cohesión entre la visión de futuro de la organización, teniendo en cuenta el desarrollo humano de las personas que la componen. Perrone y Tucker (2018), al referirse a los líderes escolares consideran que los mismos deben ayudar a los maestros a sintetizar y organizar la información proveniente de la labor educativa. En esta línea de pensamiento, Jensen (2016) describe los tres estilos de liderazgo básicos desde el contexto educativo, enfatizando su relación con el desempeño educativo, a continuación, se lista su propuesta (Acuña \& Bolívar, 2019):

- Estilo autoritario: Este estilo de liderazgo es mínimamente productivo, especialmente cuando se aplica a los alumnos, ya que se afirma que se desempeñan mejor cuando se les obliga a ser más disciplinados y centrados.

- Estilo dejar hacer: En una situación escolar, este liderazgo no tiene una influencia significativa en el rendimiento educativo de los alumnos.

- Estilo democrático: El estilo de liderazgo democrático tiene un efecto positivo en el rendimiento educativo del alumno y se encontró que es dominante en la mayoría de los directores de escuelas con alumnos de alto rendimiento.

El campo de la educación, los estudios de liderazgo han analizado preferentemente las aplicaciones y acciones del liderazgo transformacional de la dirección escolar. Por ejemplo, Leithwood y Jantzi (2005) revisan 32 investigaciones empíricas y encuentran que el estilo de liderazgo impacta de modo indirecto sobre el nivel de logro de los estudiantes y el grado de compromiso de la escuela, mediatizada la influencia por la 
cultura, el compromiso de los profesores y la satisfacción en el trabajo. Pedraja et al. (2009) establecen que el estilo de liderazgo transformacional tiene una relación directa con el logro de los estudiantes. Más recientemente, Leithwood y Sun (2012) proponen que, para mayores niveles de impacto del liderazgo, lo relevante es focalizar más en las prácticas del liderazgo que en los modelos propiamente tales. Con una muestra de 124 profesores desde educación parvularia hasta octavo grado, Fitzgerald (2015) encuentra que existe una fuerte correlación entre el liderazgo transformacional y la confianza de los profesores en la dirección; además, el estudio demuestra que la efectividad de los directores escolares se asocia a la incorporación de prácticas de liderazgo que los lleve a una relación de confianza con su plantel de profesores. No solo la confianza entre profesores y dirección se ve mejorada por el liderazgo transformacional, sino que éste impacta también en el compromiso organizacional, tal como lo muestra un estudio con 186 profesores de educación secundaria (Selamat et al., 2013). El punto a resaltar es que el liderazgo transformacional puede mejorar las instituciones de educación al generar ideas, construir y compartir una misión, compartir el poder, ganar credibilidad y generar experiencias exitosas (Yang, 2014). Grissom y Loeb (2011) plantean que el estilo de liderazgo es un aspecto clave que influye en el diseño curricular y en la enseñanza. Más aún, el estilo de liderazgo transformacional promueve el mejoramiento de las clases y del currículo, aspectos que impactan en el logro de los estudiantes; adicionalmente, las habilidades de la dirección para identificar y compartir una visión y un modelo de gestión impactan de gran manera en el nivel de logro de los alumnos. Valentine y Prater, (2011), respecto del liderazgo transaccional, un estudio con la participación de 930 profesores del norte de Israel muestra que la satisfacción en el trabajo del profesorado disminuye con los sistemas de monitoreo que le quitan autonomía en sus labores, a diferencia de lo que ocurre con el liderazgo transformacional que sí se relaciona positivamente con la satisfacción de los profesores (Bogler, 2002). Por su parte, Ibrahim y Al-Taneiji (2012), realizan una investigación en 34 escuelas de Dubai y encuentran una relación entre el lideraz- go transformacional y la efectividad de los directores; sin que dicha relación fuese estadísticamente significativa para el caso del liderazgo transaccional. Por consiguiente, la mayoría de las investigaciones sobre liderazgo y logro de los estudiantes han encontrado una relación con el liderazgo transformacional, más que con los otros estilos: 
transaccional y Laissez Faire, de la teoría de Bass (1985). (Pedraja R., Rodríguez P., Araneda G., \& Rodríguez M., 2016).

En el trabajo denominado Liderazgo directivo exitoso en la escuela secundaria. concluyen (Brito., Chagoyán, \& Herrera, 2016):

No podemos afirmar que las características y estrategias identificadas en la evidencia empírica sean 'los elementos sustanciales de toda gestión directiva de educación secundaria'. Si bien hay una serie de prescripciones de cuáles deben ser los fines de la gestión escolar (y directiva) y en ese sentido sus resultados tangibles, hay otros que no pueden ser prescritos, al menos no en el sentido estricto; sino que más bien son establecidos en torno a la cultura en que están inmersos; así, el factor contextual es un elemento de referencia que sitúa a tales o cuales estrategias como pertinentes o no y a tales o cuales procesos y resultados como deseables o no. Con base en el sustento empírico derivado del trabajo de campo, se identifica que no es la gran preparación profesional de la directora lo que genera que este tipo de comunidad escolar se movilice hacia el logro de los propósitos educativos, sino la habilidad de ésta para acompasar las demandas internas y externas: poner en juego la capacidad para generar empatía al interior del centro educativo, sensibilidad para percibir y atender los sucesos que se presentan; relaciones interpersonales neutras pero exhortativas; ser motivador natural; potenciar los liderazgos de los otros; crear ambientes en los cuales la comunidad escolar (profesores, alumnos, padres de familia, personal de apoyo) se sienta escuchada e incluida.

Por otra parte, otros autores expresan: se puede señalar que, en general, la mayor dificultad para avanzar de forma consistente y coherente en una estrategia de fortalecimiento del liderazgo educativo, radica en la desarticulación, descoordinación y fragmentación de la serie de iniciativas que afectan la función directiva, lo cual da cuenta, en algún sentido, de la inexistencia de una política explícita al respecto; se les solicita proactividad, por una parte, pero se les limita la autonomía en decisiones fundamentales; se les pide concentración en los temas docentes, pero se les satura de exigencias administrativas a veces sin sentido; se fomenta su responsabilización por resultados y rendición de cuentas, pero a la vez se les obliga a invertir tiempo y preocupaciones en implementar programas que han sido concebidos fuera de la escuela, etc. Una alternativa que está a la mano y que puede permitirnos despejar el camino se encuentra en los planes 
de mejoramiento educativo que hoy ejecutan al menos 7.000 escuelas subvencionadas del país. Si toda o gran parte de la política educativa se concentrara en garantizar que los establecimientos cuenten con una planificación estratégica y operativa, con metas claras y mecanismos idóneos para su ejecución, con buenos apoyos cuando lo requieran, con financiamiento recurrente para hacer viable su ejecución, con una rendición de cuentas financiera y técnica con consecuencias, y con las condiciones de tiempo y atribuciones necesarias, entonces no habría espacio para el desinterés y la omisión, siendo este el ambiente propicio para el florecimiento de liderazgos fuertes y comprometidos, que puedan conducir a sus comunidades educativas en grandes escuelas líderes del mejoramiento educativo. (Cancino \& Vera, 2017)

En la investigación llamada El liderazgo del director escolar: ¿Influye en la calidad educativa de la escuela secundaria?, concluye que:

La teoría llevada a la práctica dentro de las instituciones educativas del nivel secundaria, no siempre resulta coherente con la realidad. Debido a que las circunstancias del contexto en las que se lleva a cabo el proceso educativo, influyen de manera determinante, ya sea de forma positiva o negativa para el desarrollo de ésta. También es cierto que no todos los implicados en las actividades comprenden el trabajo de la misma manera, o consideran que el entendimiento y la comunicación se realizan adecuadamente para trabajar colaborativamente con un objetivo en común. Cuando se recopilaron las percepciones del colectivo docente para con sus directivos, estos consideran que su participación no siempre es la adecuada o que su acción no impacta positivamente en la gestión escolar de la institución. Por otra parte, se observó que algunas de sus respuestas se contradicen. De ahí que fue necesario analizar los factores que influyen para que se presenten estas opiniones. Infiriendo que existe una brecha o rompimiento en la percepción de los docentes con el nivel de autoridad, dejando denotar que la comunicación entre ambos actores es deficiente.

La eficacia de la institución se mide con base en los progresos alcanzados con respecto a sus puntos de partida o problemáticas presentadas con anterioridad. Un ejemplo claro de esto es la escuela A, la cual considera que su eficacia institucional ha manifestado avances significativos, dado que en ciclos anteriores la institución se encontraba en un ambiente de vandalismo y narcotráfico, donde hubo ocasiones en las que se requirió el apoyo de la 
fuerza pública (granaderos) para contener los conflictos que traspasaron la barrera del área escolar (escuela) (Gamboa, 2017).

Así mismo, en esta institución la figura del subdirector subsana algunas deficiencias directivas, favoreciendo el entendimiento del trabajo que implique al colectivo docente. Si bien es cierto, el director se debe apoyar de los recursos humanos a su disposición y delegar responsabilidades, pero sin dejar de lado sus obligaciones al grado de desentenderse de sus funciones, esto se refuerza con lo estipulado por Manés (2008), pues el director puede delegar la autoridad, pero no así su responsabilidad. Los directivos consideran que la forma en la que se dirigen al colectivo docente, es de manera fluida y no existen problemas de interpretación o favoritismo, debido a que presentan una buena comunicación. Sin embargo, los docentes opinan lo contrario, ya que la mayoría de sus respuestas coinciden en que se muestra un alejamiento verbal con el director. Externando que raras veces acuden a él, en busca de ayuda para resolver alguna problemática en la cual se presentan inmersos.

Con respecto a la escuela $\mathrm{B}$, las condiciones sociales y el poder adquisitivo de la población aledaña son favorables, lo cual facilita el proceso de enseñanza aprendizaje de la institución. Sin embargo, el grado de confort de los docentes puede llegar a sobrepasar las funciones directivas. Es aquí donde el liderazgo democrático toma fuerza al momento de negociar para la toma de decisiones, dando razones de peso, que persuadan, pero al mismo tiempo favorezcan al colectivo docente. Ahora bien, los avances y logros que se han logrado alcanzar como institución son dignos de admirarse, considerando que es una escuela relativamente nueva con sólo seis años de creación; quedando mejor posicionada que otras escuelas más longevas.

Mientras tanto, la escuela $\mathrm{C}$, presenta la misma situación que la escuela $\mathrm{A}$, donde las respuestas del director y maestros se contradicen respecto a la relación y comunicación existente entre ellos. Debido a que, en sus respuestas, el director refleja una actitud humanitaria situación que quedó de manifiesto en los resultados antes mencionados, fortaleciendo nuevamente la separación que existe entre docentes y directivos. Por lo que se refiere al desempeño directivo, resulta incongruente que por normatividad se exija una profesionalización que de buenos resultados en la escuela; pues al no ser designados para ocupar dicho puesto por sus méritos y competencias, además de no ser inducidos en su puesto, entonces su desempeño queda en la probabilidad que marca el acierto o el error. 
Para finalizar, mediante este trabajo de investigación se ha comprobado que los directivos sí reciben una preparación posterior a la asignación del cargo, la cual se centra en la actualización de los nuevos lineamientos o directrices que se van a aplicar en el sistema educativo. Desafortunadamente no abordan situaciones que fortalezcan su perfil como líder de la institución educativa que tienen o estará a su cargo. Con esto no se quiere decir que exista la necesidad de retirar a los directivos que no resulten funcionales en sus instituciones, porque eso afectaría sus derechos como trabajadores, pero no estaría demás trabajar en las debilidades, con la única finalidad de corregir los errores en los que se han incurrido desde el momento en que adquirieron el cargo. (Gamboa, 2017).

En la investigación: El liderazgo directivo y la gestión en el nivel medio superior del Instituto Politécnico Nacional de México: una mirada desde los estudiantes RIDE Revista Iberoamericana para la Investigación y el Desarrollo Educativo, concluyen que:

(...) En este sentido, se manifiesta la importancia y la trascendencia que tiene el liderazgo directivo para la gestión del Instituto. Sin embargo, a casi tres años del movimiento no se han tenido avances significativos para lograr el cambio anhelado en la Institución, con lo cual nuevamente se enfatiza la relevancia que cobra el liderazgo directivo para materializarlo. El liderazgo es fundamental para la transformación institucional debido a que, al trabajar de manera directa con estrategia, considerando la estructura, la cultura de la organización y, por supuesto, a las personas que la integran y que contribuyen con su conocimiento, experiencia, motivación y participación, se logra tomar las mejores decisiones de manera compartida para la organización. Asimismo, los estilos de liderazgo que han resultado más adecuados para ejercer el liderazgo directivo como estrategia para la gestión de las organizaciones, son el liderazgo transaccional y el transformacional, los cuales de acuerdo a las investigaciones empíricas señalan que se llevan a cabo de manera mutua: ambos son necesarios para la gestión de las instituciones educativas, así como también la importancia del liderazgo distribuido, entendido como el reconocimiento de que el liderazgo es compartido con otros miembros de la organización: él no es el único líder, sino que hay varios líderes en la organización, y al trabajar de 
manera conjunta se logran mejores resultados. En este sentido, el líder debe estar facultado para proporcionar empoderamiento a su equipo de trabajo y a las personas que contribuyan al mejoramiento de la organización. (...) (Reyes, Trejo, \& y Topete, 2017).

En la investigación denominada: Liderazgo educativo en el siglo XXI, desde la perspectiva del emprendimiento sostenible, concluye que:

(...) el liderazgo educativo se construye en la relación de todos los elementos expresados hasta ahora y en la interacción del sentido pedagógico con la innovación y la creatividad de sus líderes y la cohesión entre la visión de futuro de la organización, teniendo en cuenta el desarrollo humano de las personas que la componen. Se puede concluir que el liderazgo educativo tiene que ver con las personas y sus comportamientos frente a sus cualidades, características y competencias de los líderes a nivel individual, lo cual se proyecta en toda la organización educativa. En consecuencia, las competencias directivas refuerzan el liderazgo educativo representado en los líderes, y que, por ende, promueven la calidad educativa y la eficiencia en sus procesos. (Sierra, 2016)

En el trabajo denominado El rol de la gestión directiva en los resultados educativos, concluye que:

Si desde el punto de vista de las investigaciones de escuelas eficaces y de la literatura educativa, el trabajo del directivo es el segundo factor de éxito escolar, es pues conveniente recomendar que, como parte de las políticas educativas ecuatorianas, se promueva la realización de más estudios sobre el tema, y, en caso de verificarse el peso de los directivos escolares en el resultado de los alumnos de las escuelas que dirigen, diseñar programas de formación y apoyo que fortalezcan la gestión de todos los equipos directivos del país. (López I \& M., 2019)

En la investigación denomina, Director como líder de la Gestión Educativa de las Escuelas de alto desempeño pertenecientes a la Región del Bio Bio, concluye que:

Sin duda, el liderazgo del director influye en los resultados de las escuelas de altos desempeño, ¿Cómo podemos llegar a esa conclusión? dentro de las entrevistas los directores reconocen su tipo de liderazgo como Distributivo, tal como dice Murillo en el 
2006, este líder hace una diferencia importante en la calidad y los resultados del proceso escolar, dependiendo de la persona y no del centro o institución. Un "buen" directivo es aquel que es capaz de unir a la comunidad, de entusiasmarla en torno a un proyecto común, de implicar a todos en las decisiones. Además, dos de ellos utilizan un Liderazgo Combinado (Transformacional - Transaccional) una concepción originada en el campo empresarial y que se trasladó pronto al ámbito educativo. Central a esta concepción es, entre otras, la consideración de que el líder ha de articular una visión para la organización, comunicarla a los demás y lograr de ellos asentimiento y compromiso. El líder transformacional- transaccional se esfuerza en reconocer y potenciar a los miembros de la organización y se orienta a transformar las creencias, actitudes y sentimientos de los seguidores, pues no solamente gestiona las estructuras, sino que influye en la cultura de la organización en orden a cambiarla. (Contreras, 2019)

En el trabajo denominado, El liderazgo eje central de una propuesta de gestión directiva, desde una perspectiva humanista en las instituciones educativas distritales Chuniza y José Joaquín Castro Martínez, ultiman que:

(...) por lo cual se concluye que el liderazgo emerge como uno de los aspectos más importantes que en la actualidad requiere toda organización en especial para los equipos directivos de las Instituciones Educativas, el cual se construye y su estilo es propio de las necesidades de cada institución Educativa. Se reconoció la importancia de fortalecer el liderazgo, no sólo de los directivos, sino de cada uno de los agentes educativos; determinar factores motivacionales a nivel del fortalecimiento del talento humano, estímulos, para directivos, docentes, estudiantes, padres de familia y administrativos con el fin de que cada se sienta satisfecho con lo que hace, en donde sea capaz de posibilitar cada uno de los factores organizacionales : la orientación al logro, el aprendizaje organizacional, el clima y comunicación institucional, la asertividad, el trabajo en equipo, conciliar y resolver conflictos, y la participación: orientación a la comunidad y el entorno desde una óptica real y oportuna (...) (Galvis Q. \& Ramírez M., 2016).

En la investigación denominado, La importancia de la gestión y el liderazgo escolar en las instituciones de Educación Media Superior Tecnológica (CETIS y CBTIS) del estado 
de Tlaxcala expresa que, las características del liderazgo del directivo contribuyen a la eficiencia de la gestión en las instituciones de educación, no obstante, es necesario que el líder las practique para que pueda tener el reconocimiento de quienes día con día son parte de la institución que administra. El papel del liderazgo escolar en el desarrollo de la gestión de las instituciones educativas, así como en el establecimiento de estrategias de aprendizaje contribuye a la generación de una alta eficiencia educativa.

(...). Además, deberán comprender que el liderazgo educativo constituye un elemento importante para que realicen una gestión escolar eficaz, por lo que es conveniente advertir que esa interacción habrá de lograr que los miembros de la institución educativa colaboren de manera consciente y motivada en el logro de los objetivos y metas planteadas. (...) (Riveras \& Cavazos, 2015)

\section{DISCUSIÓN Y CONCLUSIÓN}

Como se ha afirmado, varios autores como la Real Academia Español, Idone, B., Pedraja, L, Rodríguez, E., y Rodríguez, P y el Ministerio de Educación y Cultura del Paraguay, definen o expresaron el concepto de líder y liderazgo. De ellos se puede decir que líder es un guía, alguien que conduce, que dirige a algo, en este caso es una institución educativa, en cambio la condición, la calidad, la capacidad y la forma con que el guía o líder lleva el grupo a la meta o finalidad constituye en el liderazgo.

Para ampliar, Idone, B. (2012, p 13-14), expresa que el líder tiene ciertas características como son; flexibilidad, misión y visión. Los tipos de líder son varios según las características del mismo.

En cambio, para Pedraja, L, Rodríguez, E., y Rodríguez, P (2016, p 749): se destacan los estilos de liderazgos: Liderazgo transformacional, Liderazgo transaccional y Estilo laissez faire:

Ahora bien, el Liderazgo educativo según Nuraan Davids y Yusef Waghid (2019) afirman que este Liderazgo escolar puede conceptualizarse como el conjunto de prácticas que despliegan los líderes de las instituciones escolares, que contribuyen a generar un sentido y visión compartida, que a su vez influye y moviliza el comportamiento de sus miembros hacia el alcance de los aprendizajes de los estudiantes y hacia el desempeño óptimo de los docentes en la sala de clases. El liderazgo educativo se construye en la relación de todos los elementos expresados hasta ahora y en la interacción del sentido 
pedagógico con la innovación y la creatividad de sus líderes y la cohesión entre la visión de futuro de la organización, teniendo en cuenta el desarrollo humano de las personas que la componen.

En relación al impacto de los Estilos de liderazgo, Acuña, A. y Bolívar, C. (2019, p35), citando a Leithwood y Jantzi (2005) encuentran que el estilo de liderazgo impacta de modo indirecto sobre el nivel de logro de los estudiantes y el grado de compromiso de la escuela, mediatizada la influencia por la cultura, el compromiso de los profesores y la satisfacción en el trabajo. Pedraja et al. (2009) establecen que el estilo de liderazgo transformacional tiene una relación directa con el logro de los estudiantes. Leithwood y Sun (2012) proponen que, para mayores niveles de impacto del liderazgo, lo relevante es focalizar más en las prácticas del liderazgo que en los modelos propiamente tales. Fitzgerald (2015) encuentra que existe una fuerte correlación entre el liderazgo transformacional y la confianza de los profesores en la dirección. El punto a resaltar es que el liderazgo transformacional puede mejorar las instituciones de educación al generar ideas, construir y compartir una misión, compartir el poder, ganar credibilidad y generar experiencias exitosas (Yang, 2014).

En cambio, Brito, M., Chagoyán, P. y Herrera, E., (2016, p5), identificaron que no es la gran preparación profesional de la directora lo que genera que este tipo de comunidad escolar se movilice hacia el logro de los propósitos educativos, sino la habilidad de ésta para acompasar las demandas internas y externas.

En resumen, englobando, según los estudios citados que el líder y el tipo de liderazgo tiene influencia en la meta de la institución educativa, el tipo de liderazgo más significo para la gestión escolar son: el liderazgo transaccional y el liderazgo transformacional.

En definitiva, existen similitudes en las investigaciones realizadas en cuanto a la importancia dada a las características del Liderazgo que debe poseer el Director a fin de lograr la eficiencia en la Gestión escolar, no obstante, también se resalta la necesidad de poseer un Perfil profesional acorde, experiencias en el ámbito docente por parte del Director y las habilidades sociales adecuadas para la resolución de conflictos que surjan en las instituciones educativas.

Así también, se enfatiza el papel del Liderazgo directivo en la Gestión educativa en relación con los resultados académicos, ya que, el buen desempeño de su Liderazgo pedagógico contribuye a la obtención de la Calidad educativa. 
En fin, el estilo de liderazgo impacta de modo indirecto sobre el nivel de logro de los estudiantes y el grado de compromiso de los profesores, padres de familia y la satisfacción en el trabajo.

\section{REFERENCIAS BIBLIOGRAFICAS}

Acuña; A. y Bolívar. C.(2019). Estilos de liderazgo de los directivos docentes y su relación con el modelo de gestión educativa. Línea de investigación Gestión y Calidad Educativa.

Colombia. http://repositorio.cuc.edu.co/bitstream/handle/11323/5172/Estilos\%20de\%20lide razgo $\% 20 \mathrm{de} \% 20 \operatorname{los} \% 20$ directivos $\% 20$ docentes $\% 20 \mathrm{y} \% 20$ su\%20relaci\%F3n $\% 20$ con\%20el\%20modelo\%20de\%20gesti\%F3n\%20educativa.pdf;jsessionid=1E2D F471FF5063C3E7319CA710CA2F92? sequence=1

Anderson, S. (2010). Liderazgo directivo: claves para una mejor escuela. Universidad Católica de Valparaíso. Revista Psicoperspectiva Vol. 9, No 2. http://www.psicoperspectivas.cl/index.php/psicoperspectivas/issue/view/13

Brito, M., Chagoyán, P.y Herrera. E., (2016). Liderazgo directivo exitoso en la escuela secundaria. Proceso entre la formación profesional y la intuición. https://dialnet.unirioja.es/servlet/articulo?codigo $=6064477$

Cancino C y Vera L. (2017). Políticas educativas de fortalecimiento del liderazgo directivo: desafíos para Chile en un análisis comparado con países OCDE https://www.scielo.br/scielo.php?pid=S010440362017000100026\&script=sci_ar ttext

Contreras, O. (2019). Director como líder de la Gestión Educativa de las Escuelas de alto desempeño pertenecientes a la Región del Bio Bio. Universidad del Desarrollo. Facultad de Gobierno Magíster en Políticas Educativas. https://repositorio.udd.cl/handle/11447/2742

Galvis, J- y Ramírez, S. (2016). El liderazgo eje central de una propuesta de gestión directiva, desde una perspectiva humanista en las instituciones educativas distritales Chuniza y José Joaquín Castro Martínez. Universidad libre. Facultad de Ciencias de la Educación. Departamento de postgrados. Maestría en educación con énfasis en gestión. Colombia. https://repository.unilibre.edu.co/bitstream/handle/10901/9758/EL\%20LIDERA 
ZGO\%20EJE\%20CENTRAL\%20DE\%20UNA\%20PROPUESTA\%20DE\%20G ESTIÓN\%20DIRECTIVA.pdf?sequence $=1 \&$ isAllowed $=\mathrm{y}$

Gamboa, M., Patiño, M,. y García, M. (2017). El liderazgo del director escolar: ¿influye en la calidad educativa de la escuela secundaria? Temática general: política y gestión de la educación y su evaluación, la ciencia y la tecnología. http://www.comie.org.mx/congreso/memoriaelectronica/v14/doc/2865.pdf

Idone, B. (2012). Estilos de liderazgo del director según percepción docente y autoevaluación del desempeño docente en una institución de Ventanilla. Perú. http://repositorio.usil.edu.pe/bitstream/123456789/1185/1/2012_Idone_Estilos\% 20de\%20liderazgo\%20de1\%20director\%20según\%20percepción\%20docente\%2 0y\%20autoevaluación\%20del\%20desempeño\%20docente\%20en\%20una\%20ins titución\%20de\%20Ventanilla.pdf

López, I. y López, M. (2019). El rol de la gestión directiva en los resultados educativos.

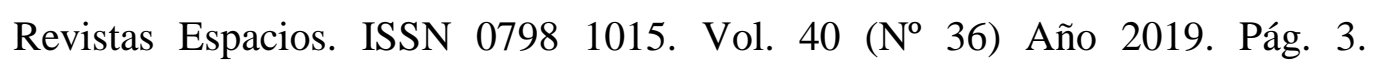
http://www.revistaespacios.com/a19v40n36/a19v40n36p03.pdf

Ministerio de Educación y Cultura. (2014). El liderazgo: características e impacto en la gestión educativa. República del Paraguay. El liderazgo: características e impacto en la gestión educativa. Programa de capacitación de los educadores para el mejoramiento de los aprendizajes de niños, niñas, jóvenes y adultos del Paraguay. https://www.mec.gov.py/talento/archivo/convocatoria072017/material/ejes/curriculum/28_curso_de_especializacion_modulo_3.pdf

Pedraja, L, Rodríguez, E., y Rodríguez, P (2016). Estilos de liderazgo de dirección escolar y el logro académico de los estudiantes: un estudio exploratorio. Interciencia, 41(11),748-756.

ISSN:

0378-1844. https://www.redalyc.org/articulo.oa?id=339/33948191004

Real Académica Española. Liderazgo. Diccionario online, https://dle.rae.es/liderazgo?m=form

Reyes, V; Trejo, M; y Topete, C. (2017). El liderazgo directivo y la gestión en el nivel medio superior del Instituto Politécnico. RIDE Revista Iberoamericana para la Investigación y el Desarrollo Educativo. E-ISSN: 2007-7467. http://www.redalyc.org/articulo.oa?id=498154006002 
Rivera, J. y Cavazos, J. (2015). La importancia de la gestión y el liderazgo escolar en las instituciones de Educación Media Superior Tecnológica (CETIS y CBTIS) del estado de Tlaxcala. Revista Iberoamericana para la Investigación y el Desarrollo Educativo. ISSN 2007 - 7467

Sierra, G. (2016). Liderazgo educativo en el siglo XXI, desde la perspectiva del emprendimiento sostenible. Revista de Escuela Administración y Negocio. No. 81. Colombia. http://dx.doi.org/10.21158/01208160.n81.2016.1562 